\title{
MULTIPLE PLANT LEAF DISEASE CLASSIFICATION USING DENSENET-121 ARCHITECTURE
}

\author{
Aswin Vellaichamy S \\ School of Electronics Engineering Vellore Institute of Technology, \\ Vellore, Tamilnadu, India \\ Akshay Swaminathan \\ School of Electronics Engineering Vellore Institute of Technology, \\ Vellore, Tamilnadu, India \\ C Varun \\ School of Electronics Engineering, \\ Vellore Institute of Technology, Vellore, Tamilnadu, India

\section{Dr. Kalaivani S} \\ Associate Professor, School of Electronics Engineering, \\ Vellore Institute of Technology, Vellore, Tamilnadu, India
}

\begin{abstract}
Agriculture is the backbone of a country in terms of economy and survival of the people. To maintain a high efficiency of crop production we look to avoid plant diseases. The proposed algorithm is to optimize the information from the resources available to us for the betterment of the result without any complexity. The neural network used for classification is the Dense Convolution Neural Network (DCNN). In this project, a pre-trained neural network model (densenet-121) which is imported from the keras library has been used for training. A convolution may be a simple application of a filter to an input that leads to activation. Frequent application of equivalent filter to an input, leads to a map of activations called feature map, indicating the locations and strength of a detected feature in an input such as an image. The convolutional networks help to automatically learn an outsized number of filters in parallel specific to a training dataset. This algorithm helps provide an efficient result in detecting plant diseases, which in turn helps the economy of the country as well. Using 35779 images from Huges DP Plant-Village dataset from Kaggle, the densenet-121 has been used to classify the 29 different diseases for 7 plants (potato, tomato, corn, bell pepper, grape, apple and cherry). In this project, the original image is converted to HSV color form and then the masked image is generated by thresholding and given to the proposed model for training and classification, giving an average accuracy
\end{abstract}


(theoretical) of $98.23 \%$. When all classes of plant disease are given together to the model for training on google colab platform, (Tesla-T4 processor) we got an average accuracy of $94.96 \%$ for 50 epochs with a learning rate of 0.002. Additionally, a basic user-friendly website to test the trained model for the disease affected plant images and get prescription for the plant disease. Further the scope to extend the dataset used for training to identify many plant diseases.

Keywords: metallic surface, VGG-16, Neural Networks, defect detection

Cite this Article: Aswin Vellaichamy S, Akshay Swaminathan, C Varun and Kalaivani S, Multiple Plant Leaf Disease Classification using Densenet-121 Architecture, International Journal of Electrical Engineering and Technology (IJEET), 12(5), 2021, pp. 38-57.

https://iaeme.com/Home/issue/IJEET?Volume=12\&Issue $=5$

\section{INTRODUCTION}

India being a cultivated country and about $70 \%$ of the population depends on agriculture. Farmers have large range of diversity for choosing various suitable crops and finding the acceptable pesticides for the respective plants. Disease on plants results in large reduction in both the standard and the quantity of agricultural products. The study of diseases asks the studies of visually observable patterns on the plants. Monitoring of health and disease on plants plays a crucial role for successful cultivation of crops within the farm. In youth, the monitoring and analysis of plant diseases were done manually by an expertise person therein the field. This requires tremendous amount of labor and also requires excessive time interval. The image processing techniques are often utilized in the disease detection. In most of the cases, the symptoms of a plant disease are seen on the leaves and stem. The plant leaf for the detection of disease is taken into account which shows the disease symptoms. In this project a variety of plants such Maize, Tomato, Potato and Capsicum. Today, the potato is the third most vital crop within the world in terms of food consumption, after rice and wheat, and people in developing countries depend on potatoes for their survival. More than 156 countries produce potatoes and quite a billion people worldwide eat them on a daily basis. Maize is one among the foremost versatile emerging crops shaving wider adaptability under varied agro-climatic conditions. Globally, maize is understood as queen of cereals because it's the very best genetic yield potential among the cereals. Tomatoes have many advantages over growing other sorts of crops, such as, their high yield which ends up in their high value. They have a high nutritional value of pro-vitamin A and C. As well as being ranked first on their nutritional contribution to a human's diet. Colored capsicum which also knows as "sweet pepper" or "bell pepper" is one of the important vegetable crops cultivated in green houses and up to some extent under the shade net house in milder climatic regions. It is rich in vitamin-A, C and minerals. As mentioned above the importance of each plant, we try to avoid the disease rate so that the cultivation of these plants becomes fruitful and helps increase the accuracy which directly proportionate to the increase in economy and reduce in wastage, by following various image processing techniques.

\section{LITERATURE SURVEY}

Various machine vision-based approaches have been pro- posed to identify different diseases, pests and stress in various variety of crops over the recent years. Anamietal. [1] have used Sequential Forward Floating Selection (SFFS) to reduce the overlapping between the color features for detecting paddy diseases and stresses using classifier models such as Support Vector Machine (SVM), Back Propagation Neural Network (BPNN) and k -Nearest Neighbor (k-NN) and compared their accuracies .Muppala et al. [2] proposed a four- layer deep neural network 
with search and rescue optimization (DNN-SAR) pest detection method was proposed to identify yellow stemborer and leaf folder moths in the paddy field. Sethy et al. [3] and Kartikeyan, Pet al. [4] have compiled all image processing techniques used to detect plant diseases over the years 2007-2018. These papers were very useful for testing many current image processing techniques. Ramesh et al. [5] used a dataset containing 575 images for paddy disease detection where Classification of diseases is carried out by Optimized Deep Neural Network with Jaya Optimization Algorithm (DNN-JOA). Khan et al. [6] have proposed a method combined of color balancing, k-means clustering, super pixel operations, Histogram of Gradients (HOG), Grey Level Co- occurrence Matrix (GLCM) features and Random Forest (RF) technique to classify tomato diseases. Their data set consists of both real time and downloaded dataset. Mathew al et. [7] developed a novel neural network model to classify fungal diseases in banana using image enhancement, segmentation and extracting feature vectors using DWT (Discrete Wavelet Transform), DTCWT (Dual Tree Complex Wavelet Transform) and Ranklet transform and then compared with elliptical local binary pattern (ELBP), MeanELBP and MedianELBP. As for Convolution Neural Network (CNN) Rahman et al. [8] and Sharma et al. [9] have established a CNN model to classify paddy diseases using segmented and full images. Bakar et al. [10] has worked on classifying rice plant diseases over three categories by multi-level thresholding after converting live images to HSV color format. Also, the same type of network is seen being used in [11] for developing a smart agriculture model. Considering the neural network Deep Con- volution Neural Network (DCNN), [12] have used the same neural network model trained to classify corn plant disease and had achieved an accuracy of $88.46 \%$. A CNN model was developed to identify 3 different maize diseases in [13]. The blackleg detection in potato was developed using ResNet-18, which is an established CNN model, by Manya et al. [14]. Potato disease detection was modelled using VGG19, a established CNN model was discussed in [15]. A Multi-class SVM based classifier is used for identifying tomato disease in [16]. A tomato disease classifier was developed by Santhosh et al. [16] using CNN model. Other neural networks like back propagation neural network (BPNN) is used in [17] where 24 texture features are extracted through GLCM for classifying potato disease detection. Going by the image dataset used the feature set based multi-diseases classification (FSMDC) [18] had the same dataset [32] we have used to test in our model, where they worked with uniquely extracted feature set. SVM, MLP and LR techniques are used to compare with various other state of art techniques. As for the image processing technique, they used a model to make mask for images by region growing and have shown an average accuracy of $93.2 \%$. Iyyanaret al. [19] have made a model to classify distinct disease in rice, tomato and potato using a CNN network. Jadhav et al. [20] has made a review on the advancements in detecting plant and vegetable diseases. In [21] HSV based apple disease classification using machine learning was performed and compared with other color spaces. Ghorai A. K. et al.[22] has reviewed all major diseases and deficiencies of crops along with various machine learning techniques used in the years. Patil et al. [23] has analyzed and complied 16 existing image processing and classification techniques for pomegranate disease detection. We have taken their data for comparison with our model. As for the neural architecture all information is referred from [26, $27,28]$ for dense net model as a whole is analyzed and explained. Islam et al. introduced an AI based plant sickness analysis approach (MLPDD) [18] to categorize illnesses on potato plants. Patil P et al. [29] looked over an automated crop disease management technique (ACDMT) for potato. A k-means clustering-based semi-automatic technique (KMCSAT) was introduced by Kaur et al. [31] for plant illness order. In our work, we have used conventional RGB to HSV conversion, similar to which is used in $[4,21]$ and further thresholding the H-region to extract important boundary features of the leaf. Thereafter developing the binary mask, this in turn is applied to the original image to remove unnecessary background and given into our neural network for analysis and classification. 


\section{PROPSED METHODOLGY}

Pre-processing techniques are used to improve image quality and image features for any field of application. Plant disease detection is no exception. Images in the dataset have background information which is unnecessary for disease detection in leaves of crops. This is overcome by RGB color form image to HSV form, segmenting required portion of image by histogram based thresholding and hole-filling ending into a binary mask image and then masked by dot multiplication on the binary mask, finally resizing it for neural network training. A quick overview is given below in the flowchart and each part is elaborated in the upcoming headings. The below fig. 1 has the flow of work simplified through the flowchart.

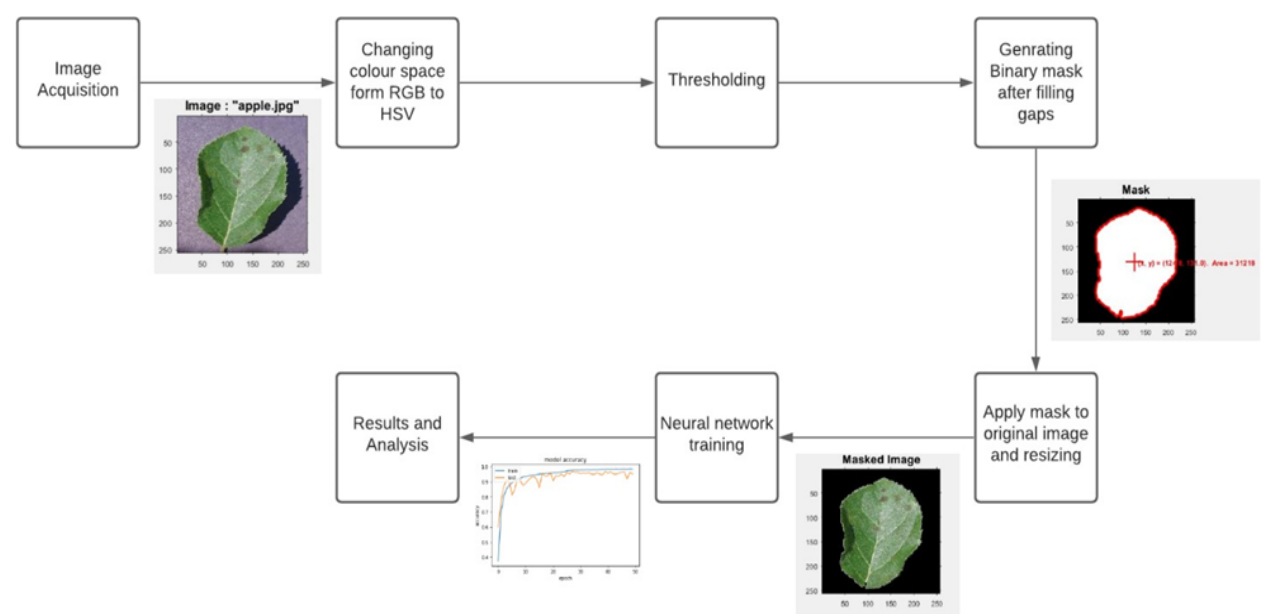

Figure 1 Plant Village dataset images

\subsection{Image Accquistion}

We have used a downloaded dataset for caring out the training our neural network model and have extended for testing real time images via beta version website. We have taken 35779 images of size 256 × 256 from PlantVillage (Huges DP) dataset [32] which is available in Kaggle across 29 labels of diseases for 7 plants namely apple, tomato, corn, bell pepper, grape, cherry and potato. The images used for each category are displayed below in fig.2. Table 1 gives the image count for each category under various crops.
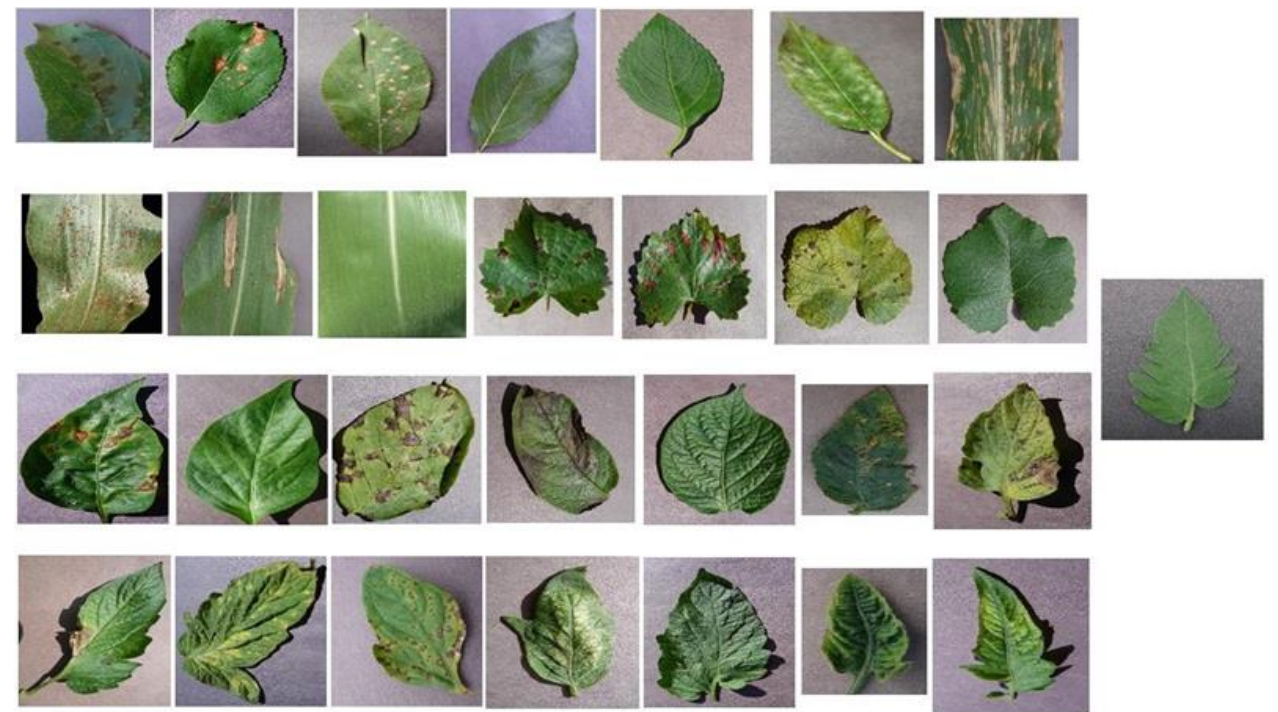

Figure 2 Plant Village dataset images 
Aswin Vellaichamy S, Akshay Swaminathan, C Varun and Kalaivani S

Table 1 Total no.of images for each class of disease

\begin{tabular}{|c|c|c|}
\hline Crop Name & Image category & Number of Images \\
\hline Apple & \begin{tabular}{|l} 
Scab \\
Black rot \\
Healthy \\
\end{tabular} & $\begin{array}{c}630 \\
621 \\
1645 \\
\end{array}$ \\
\hline Cherry & $\begin{array}{l}\text { Cedar apple rust } \\
\text { Powdery mildew } \\
\text { Healthy }\end{array}$ & $\begin{array}{c}275 \\
1052 \\
854\end{array}$ \\
\hline Corn & $\begin{array}{l}\text { Cercosporin leaf spot } \\
\text { Common rust } \\
\text { Northern Leaf Blight } \\
\text { Healthy }\end{array}$ & $\begin{array}{c}513 \\
1192 \\
985 \\
1162 \\
\end{array}$ \\
\hline Grape & $\begin{array}{l}\text { Black rot } \\
\text { Esca (black measles) } \\
\text { Leaf blight } \\
\quad \text { Healthy }\end{array}$ & \begin{tabular}{ll}
\multicolumn{2}{c}{1180} \\
1384 \\
$1076^{423}$
\end{tabular} \\
\hline Pepper-bell & $\begin{array}{l}\text { Bacterial spot } \\
\text { Healthy }\end{array}$ & $\begin{array}{c}997 \\
1478 \\
\end{array}$ \\
\hline Potato & $\begin{array}{l}\text { Early Blight } \\
\text { Late Blight } \\
\text { Healthy }\end{array}$ & $\begin{array}{c}1000 \\
1000 \\
152\end{array}$ \\
\hline Tomato & \begin{tabular}{|l|} 
Bacterial spot \\
Early blight \\
Late blight \\
Healthy \\
Leaf mold \\
Septoria leaf spot \\
Spider mites \\
Yellow leaf curl virus \\
Target spot
\end{tabular} & $\begin{array}{c}2127 \\
1000 \\
1909 \\
1591 \\
952 \\
1771 \\
1676 \\
5357 \\
\\
1404\end{array}$ \\
\hline
\end{tabular}

\subsection{Image Pre-Processing}

We start with converting colour form of the RGB image HSV. The procedure for this is given below:

The R, G and B values are divided by 255 to change the range of values from $0-255$ to $0-1$.

$$
\begin{gathered}
\mathrm{R}^{\prime}=\mathrm{R} / 255 \\
\mathrm{G}^{\prime}=\mathrm{G} / 255 \\
\mathrm{~B}^{\prime}=\mathrm{B} / 255 \\
\mathrm{C}_{\max }=\max \left(R^{\prime}, G^{\prime}, B^{\prime}\right) \\
\mathrm{C}_{\min }=\min \left(R^{\prime}, G^{\prime}, B^{\prime}\right) \\
\Delta=\mathrm{C}_{\max }-\mathrm{C}_{\min }
\end{gathered}
$$

Hue calculation:

$$
H=\left\{\begin{array}{l}
60^{\circ} \times \frac{\mathrm{G}^{\prime}-\mathrm{B}^{\prime}}{\Delta} \bmod 6, \mathrm{C}_{\max }=\mathrm{R}^{\prime} \\
60^{\circ} \times \frac{\mathrm{B}^{\prime}-\mathrm{R}^{\prime}}{\Delta}+2, \mathrm{C}_{\max }=\mathrm{G}^{\prime} \\
60^{\circ} \times \frac{\mathrm{R}^{\prime}-\mathrm{G}^{\prime}}{\Delta}+4, \mathrm{C}_{\max }=\mathrm{B}^{\prime}
\end{array}\right.
$$

Saturation calculation:

$$
S= \begin{cases}0 & \text {,if } C_{\max }=0 \\ \frac{\Delta}{C_{\max }} & \text {,if } \mathrm{C}_{\max } \neq 0\end{cases}
$$

Value calculation:

$$
V=\mathrm{C}_{\max }
$$


Where, R, G and B represent values of red, green and blue in original image and $\mathrm{H}, \mathrm{S}$ and $\mathrm{V}$ represent hue, saturation and value after processing the original image. In the Fig.3 we have displayed the sample images which have been converted to HSV color plane and also their plane wise representation.

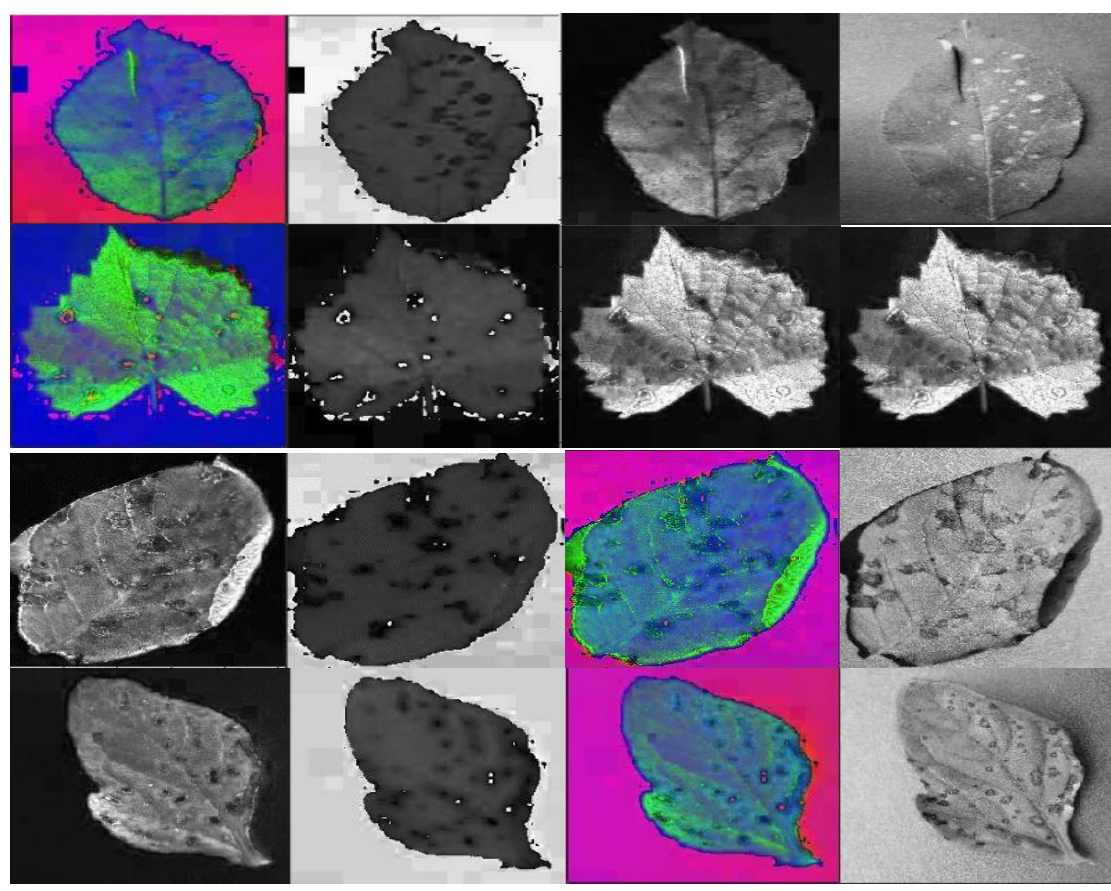

(a)

(b)

(c)

(d)

Figure 3 The first row shows the apple cedar rust leaf, the second row shows the grape black rot leaf, the third row shows the potato early blight leaf and the last row shows the tomato septoria leaf spot.

(a) HSV image, (b) H-plane, (c) S-plane, (d) V-plane.

\subsection{Image Segmentation}

Generating binary mask via thresholding image is defined as:

$$
\begin{gathered}
g(x, y)=1, \mathrm{~T} 1 \leq \mathrm{f}(\mathrm{x}, \mathrm{y})<\mathrm{T} 2 \\
0, \text { otherwise }
\end{gathered}
$$

Where $\mathrm{x}$ and $\mathrm{y}$ represent the coordinal values of the pix in image $g$ and $\mathrm{f}$. Thresholding is done where $\mathrm{T} 1$ is equal to 0.130 and $\mathrm{T} 2$ at 0.600 for $\mathrm{H}$-values alone. These values are chosen after statistical analysis of images and their histograms. After this step there are still discrepancies in the image due to noise and shadows, which makes the binary map imperfect. To overcome this, we fill these gaps by morphological reconstruction. After this, we get the binary mask. The area of the binary mask is highlighted by a red line along with the identification of the centroid of the image.

The obtained binary mask is then used to make masked image by,

\section{Masked image $=$ binary mask. * Original image}

Where, “*” represents normal dot multiplication of corresponding values that are present in binary mask and original image. These have been displayed the sample outputs of images obtained in each of the above-described steps below. These masked images are then resized to 64 to reduce time consumption for neural network training. 


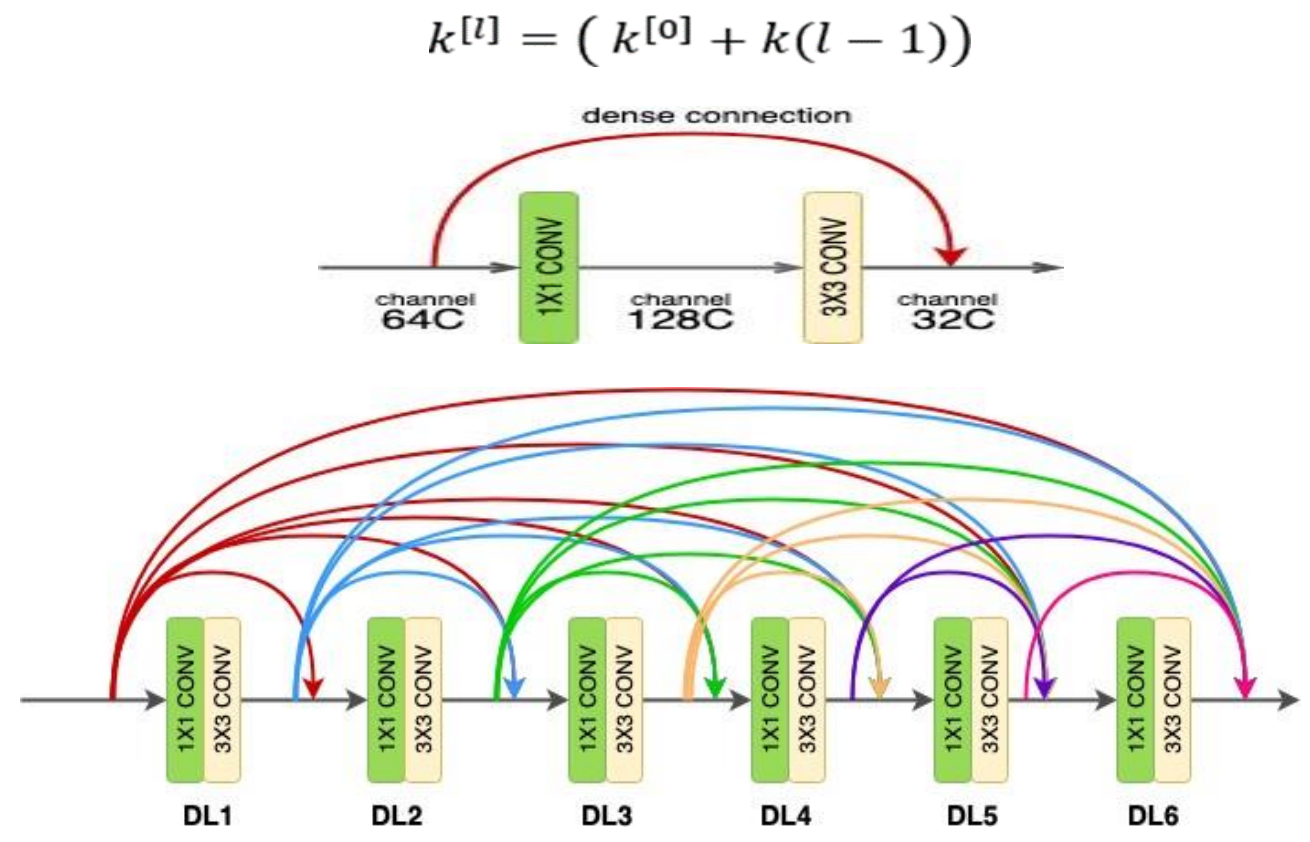

Figure 4 Densenet connection block (up), dense connected layer(down)

\section{FEATURE EXTRACTION WITH DCNN}

The neural network used here is a densenet-121 a type of dense convolution neural network. DenseNet (Dense Convolutional Network) is a design that centers around making the deep learning networks go much more profound, and yet making them more effective to prepare, by utilizing more limited associations between the layers. DenseNet is a convolutional neural organization where each layer is associated with any non-subsequent layers that are more profound in the organization, that is, the principal layer is associated with the second, third, fourth etc., the subsequent layer is associated with the third, fourth, fifth, etc. This is done to empower greatest data stream between the layers of the organization. To protect the feedforward nature, each layer acquires contributions from every one of the past layers and gives its own element guides to every one of the layers which will come after it. So, the ' $i$ th' layer has 'I' data sources and comprises of highlight guides of all its first convolutional blocks. Its own element maps are given to all the following 'I-I' layers. This presents ' $\left(I^{*}(I+1)\right) / 2^{\prime}$ associations in the organization, as opposed to simply 'I' associations as in customary profound learning models. It subsequently requires fewer boundaries than customary convolutional neural organizations, as there is no compelling reason to learn insignificant element maps.

The number 121 in densenet-121 is computed as follows:

DenseNet-121: $-5+[2 \times(6+12+24+16)]$

5-Convolution and Pooling Layer

3- Transition Layers $(6,12,24)$

1- Classification Layer (16)

2- Dense Block $(1 \times 1$ and $3 \times 3$ conv $)$

DenseNet comprises of two significant units other than the essential convolutional and pooling layers. Those are the Dense Blocks and Transition layers. The quantity of filters changes between the DenseBlocks, expanding the components of the channel. The development rate $(\mathrm{k})$ helps in generalizing up the $\mathrm{l}^{\text {th }}$ layer. It controls the measure of data to be added to each layer. In simple terms, it is basically the number of channels output by a dense block. This means that the number of features received by a dense layer (l) from its preceding dense layer (1-1) is $\mathrm{k}[1]$. This is referred to as the growth rate because after each layer, $\mathrm{k}[1]$ channel features 
are concatenated and fed as input to the next layer. A dense layer consists of 2 convolutional operations $-1 \times 1$ CONV (conventional convolution operation for extracting features) and $3 \mathrm{x}$ $3 \mathrm{CONV}$ (bringing down the feature depth/channel count). The DenseNet-121 consists of 6 such dense layers in a dense block. The below fig. 4 gives a pictorial representation of the dense layer and dense block. The depth of the output of each dense block is equal to the growth rate of the dense block.

DenseNet begins with a fundamental convolution and pooling layer. After that there is a dense block layer followed by a progress layer, another dense block layer followed by a change layer, another dense block followed by a progress layer, lastly a thick square followed by a characterization layer. Each dense block has two convolutions, with $1 \times 1$ and $3 \times 3$ estimated pieces. In dense block 1 , this is done multiple times, in dense block 2 it is done multiple times, in dense block 3, 24 times lastly in dense block 4, 16 times. Each dense- layer consists of 2 conv operations $-1 \mathrm{X} 1 \mathrm{CONV}$ (conventional conv operation for extracting features) and $3 \mathrm{X} 3$ CONV (bringing down the feature depth/channel count).

At the end of every dense block, the number of feature-maps accumulates to a value of -

Input features + (number of dense layers $x$ growth rate)

To bring down this channel count, a transition layer or transition block is added between two dense blocks. The transition layer (fig.5) consists of -

$1 \times 1$ CONV operation

$2 \times 2$ AVG pooling operation

The $1 \times 1$ CONV operation reduces the channel count to half.

The $2 \times 2$ AVG pooling layer is responsible for down-sampling the features in terms of the width and height.

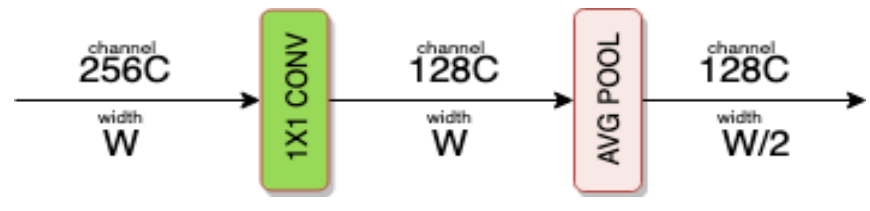

Figure 5 Transition block

The full network flow figure is shown below in fig.6[27]. The neural network model has a total of 7.3125621 million parameters evaluated for classification of images, wherein 7.226353 million are trainable and the rest 86,208 being non-trainable. The learning rate is set to 0.002 and 437 iteration for each epoch, for a total of 50 epochs in our model. The model has been set to take $75 \%$ (approx.) of images under each label for training and the remaining 25\% (approx.) for validating. The python output for the model is shown in the below fig.7.

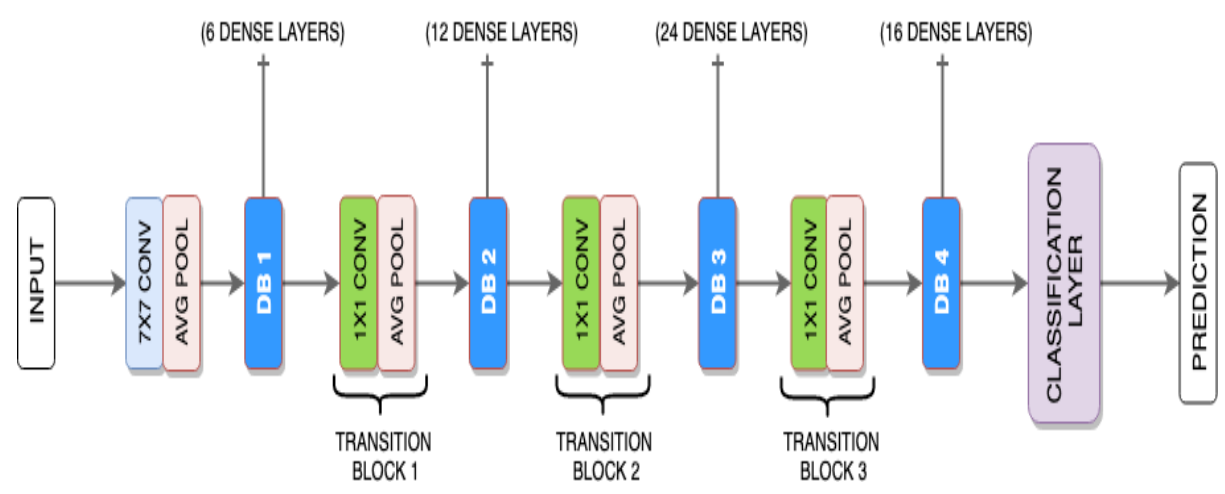

Figure 6 Full dense network 


\section{RESULTS AND DISCUSSION}

Performance is evaluated for the proposed image processed neural network-based classification technique done on PlantVillage dataset. The dataset is susceptible uniform data availability which affects the classification results. Training of neural model with downloaded dataset gives less accuracy as compared to live images since many image parameters are not monitored like lighting, clarity, etc. Accuracy is calculated as the end- result of the neural network training through the below:

$$
A=(\text { No. of }(Y \mathrm{P}==Y \mathrm{c}) /(\text { Total no. of images used })) * 100
$$

Here $Y \mathrm{P}$ is the image label which is obtained via accessing folder labels before they are given into the training and $Y \mathrm{c}$ is the image labels predicted by the neural network. All accuracy values are calculated at the end of neural network training. We have qualitatively and quantitatively analyzed the results and reported below as follows. The visual illustration of segmentation results for the apple images dataset, which includes healthy, apple scab, and black rot are shown in fig.7. The apple leaves affected by scab become puckered or twisted and have black, circular spots on their upper surface. The signs of black rot are purple spots on the upper part with centers turn tan, yellowish-brown, or brown. The next is cherry leaf images for healthy and powdery mildew categories (fig.7). The symptoms of cherry powdery mildew are light circular nature powdery looking patches on leaves. Corn leaf images for corn cercospora grey leaf spot, corn common rust, and corn northern leaf blight category are displayed in the (fig.8). The Cercospora leaf spot having a faint watery halo that appears tan to brown in the color of rectangular shape and bordered by the veins of the leaf. The corn common rust spores are brick red and orange in color. The northern leaf blight shows symptoms of large, usually oval, light brown, or grey leaf spots, sometimes it shows long darker margins. The grape leaf images for healthy, black rot, leaf blight (Isariopsis leaf spot), and grape esca (black measles) class. The black rot shows small, brown circular lesions on grape leaves. Leaf blight also named Isariopsis leaf spot having scattered, somehow angular, purple-brown spots on the upper surface of the leaf. The grape esca (black measles) symptoms comprise shoot tip dieback along with leaf discoloration. The bacterial leaf spot causes a dark, purple-brown spot with a light brown center.

Table 2 DCNN Network Filter and Parameter in each layer

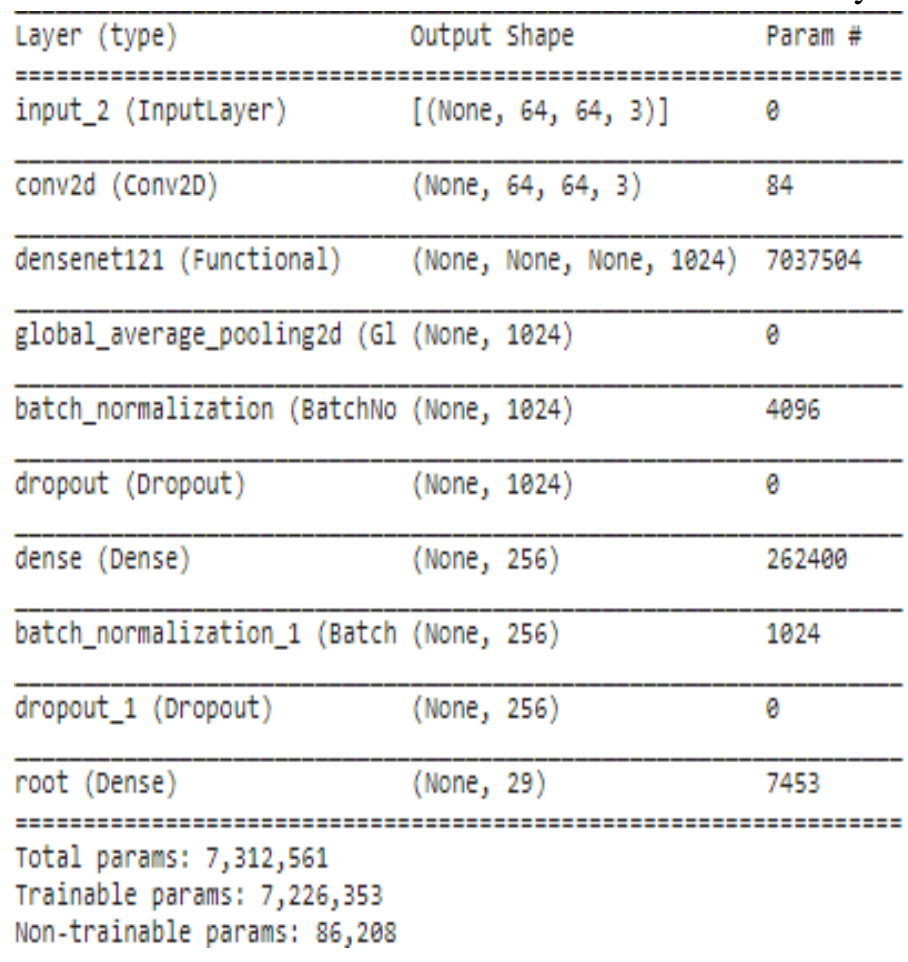


Sample images of potato dataset containing healthy, early blight, and late blight is given below (fig.9). The early blight leaves have small brown or black, it may enlarge having a yellow halo. The late blight appears as wet spots, generally at the edges or tips of lower leaves where the water or dew tends to accumulate. (Fig.10), shows exclusively all the tomato images collected. For tomato bacterial spot, dark brown, oval to elongated spots; coalesce to streaks; considerable loss of lower leaf foliage can occur. The tomato early blight on stems appears as lesion with dark border and gray center which can encompass and girdle stem causing death of lower canopy leaves. The tomato Septoria leaf spot has dark elongated spots develop on petioles and stems leading to loss of lower canopy, and unthrifty plants. Tomato Late blight is newer genotypes of late blight are more aggressive, especially to stems. Tomato mosaic virus disease is stunted and shows a great number of black streaks on leaves, stems and discoloration on fruit. Though major labels (type of plant) are classified correctly, there is a small fraction of error in detecting minor classes (type of disease).
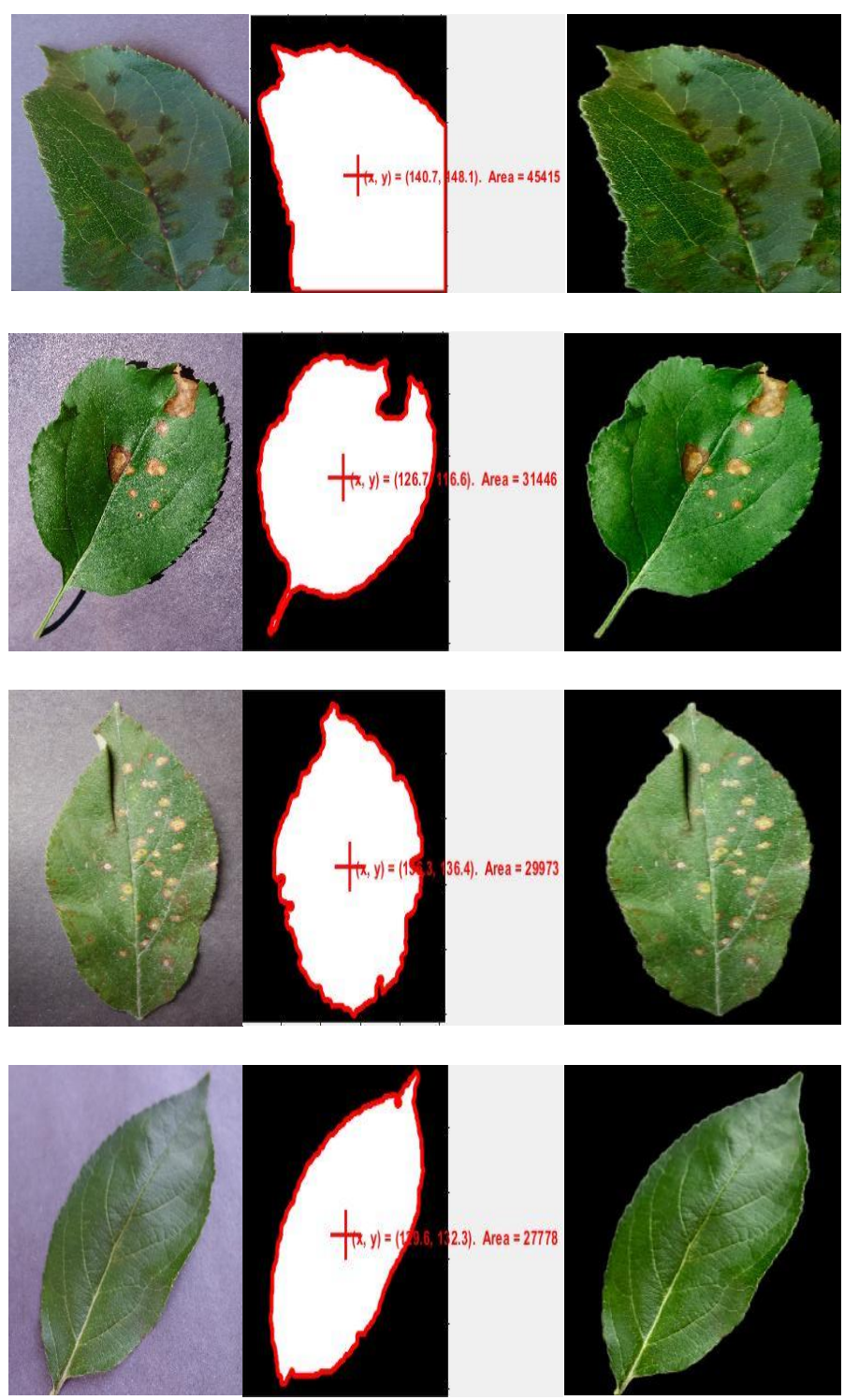
Aswin Vellaichamy S, Akshay Swaminathan, C Varun and Kalaivani S
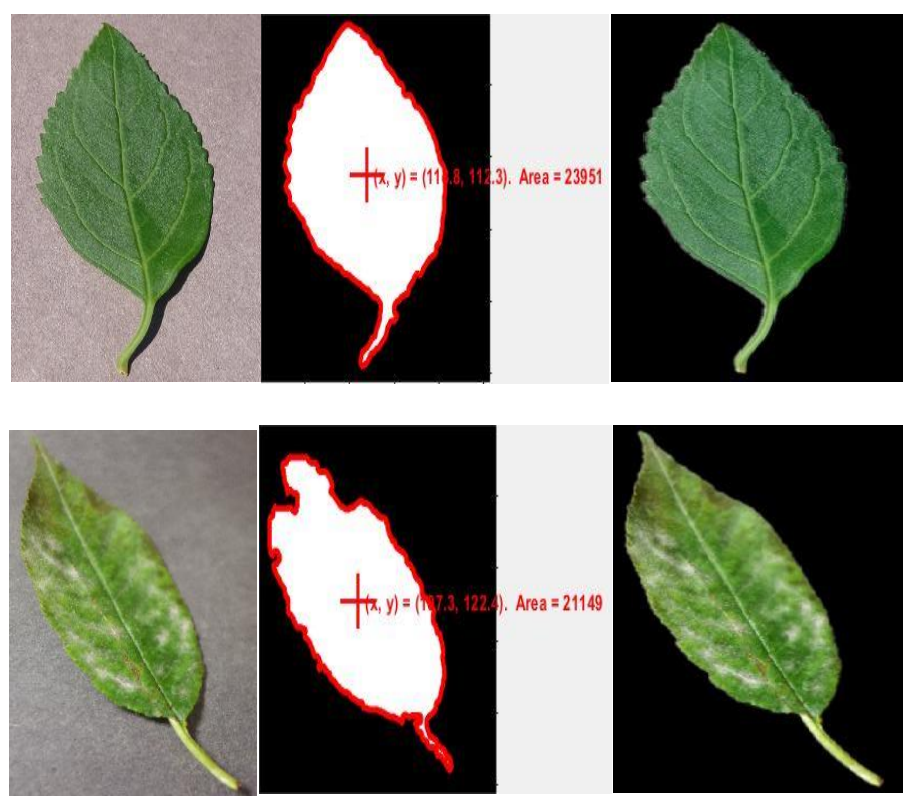

(a)

(b)

(c)

Figure 7 The first four rows show the apple leaves (scab, black rot, cedar apple rust and healthy), the fifth and sixth rows show the cherry leaves (healthy and powdery mildew). (a) original images, (b) Binary masked images, (c) Final masked images.
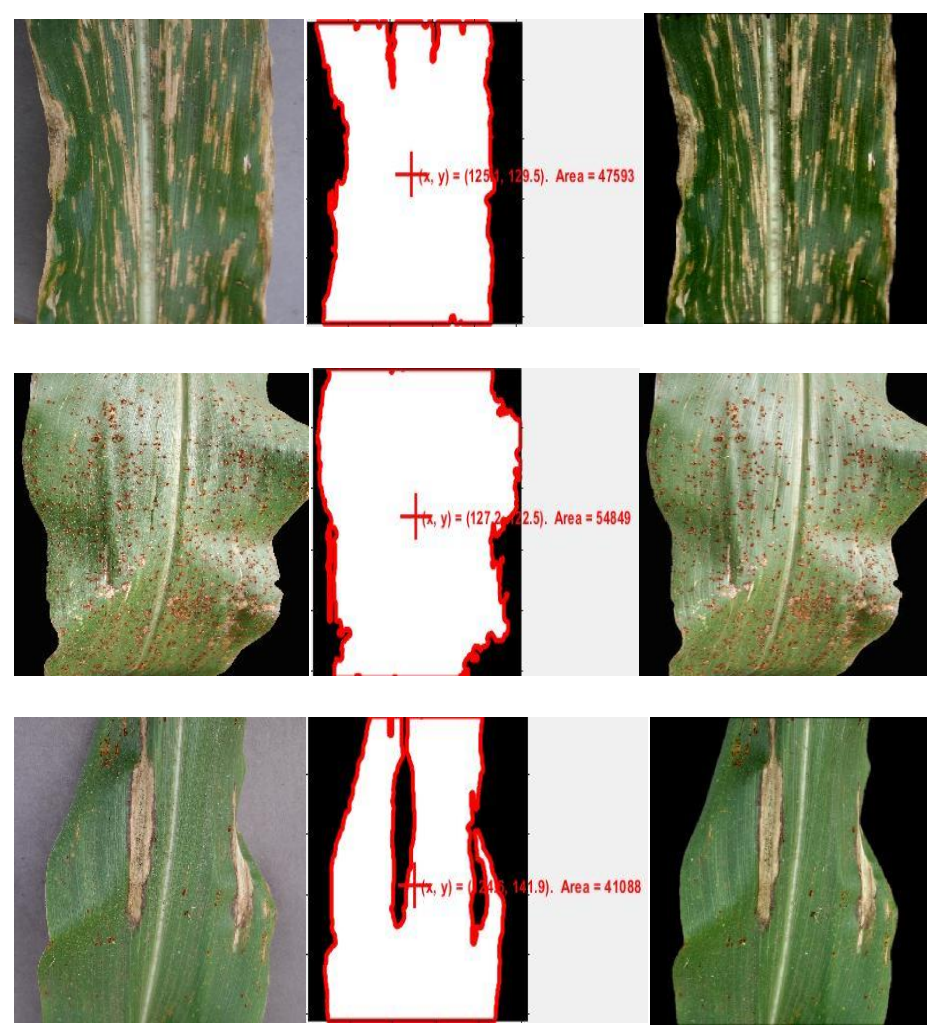

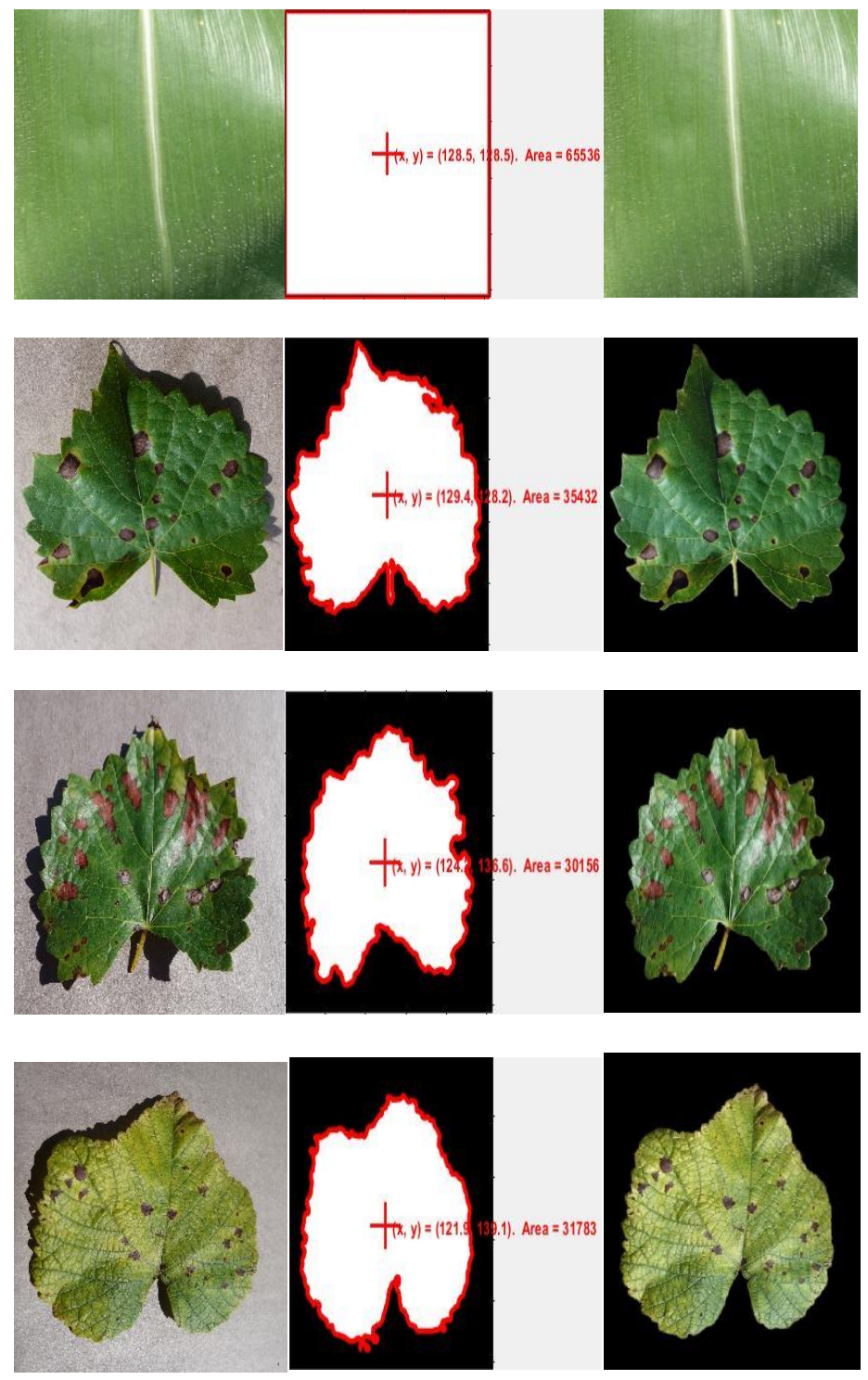

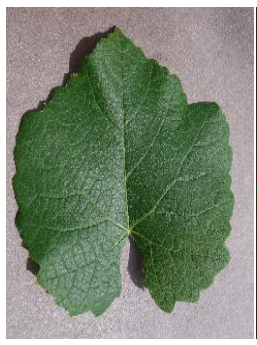

(a)

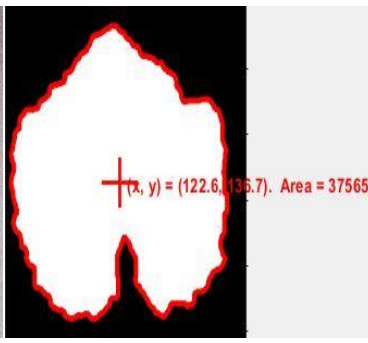

(b)

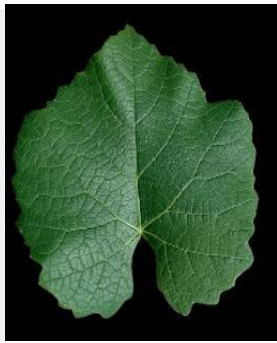

(c)

Figure 8 The first four rows show the corn leaves (cercospora leaf spot, common rust, northern leaf blight and healthy), the fifth - eighth rows show the grape leaves (black rot, black measles, leaf blight, healthy). (a) original images, (b) Binary masked images, (c) Final masked images. 
Aswin Vellaichamy S, Akshay Swaminathan, C Varun and Kalaivani S
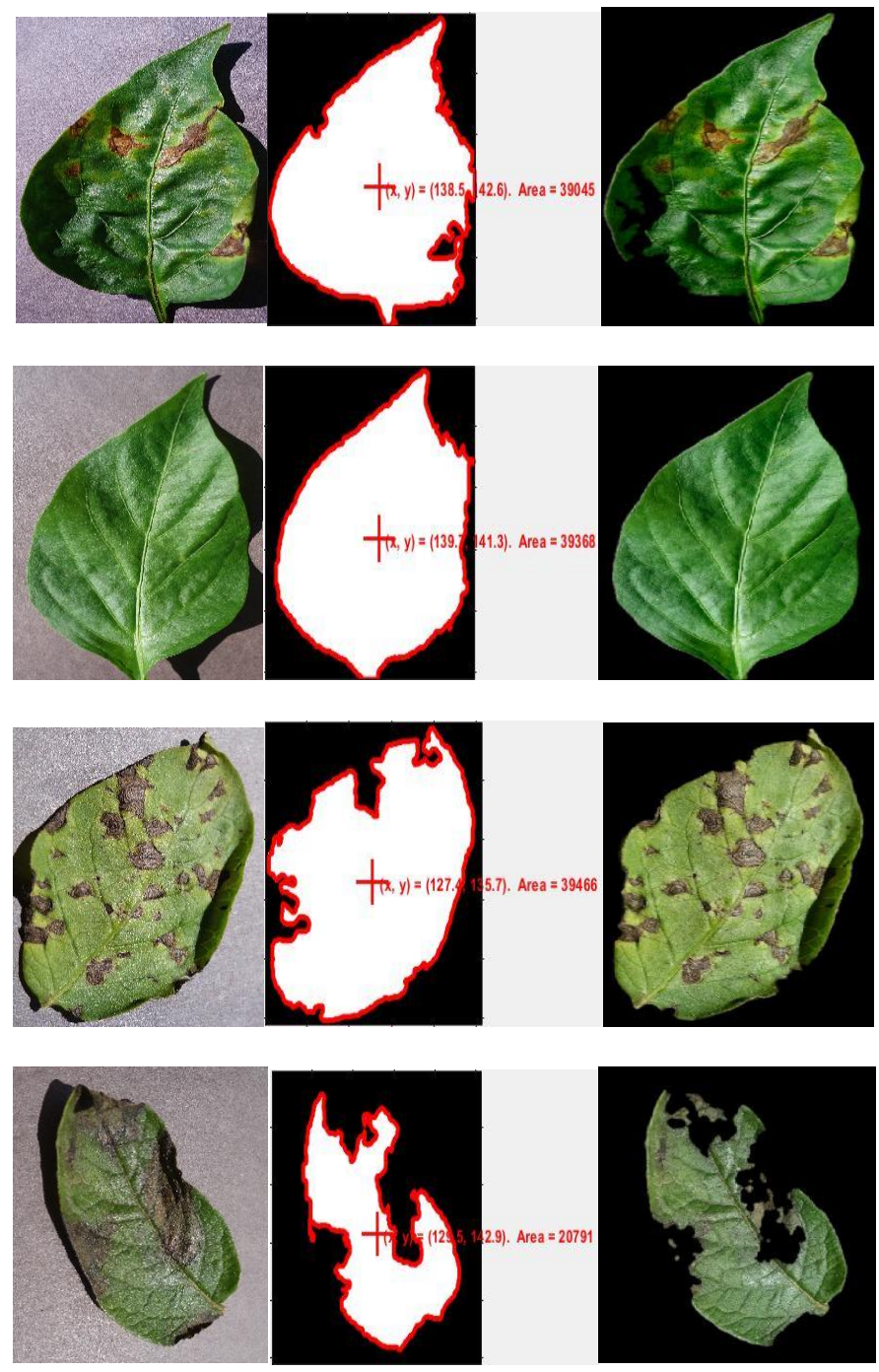

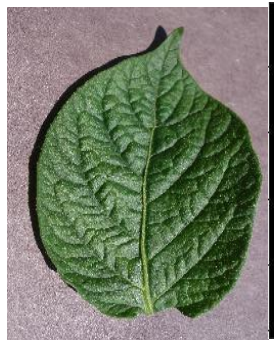

(a)

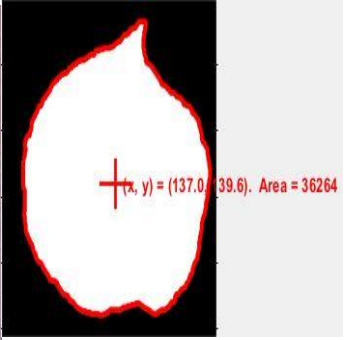

(b)

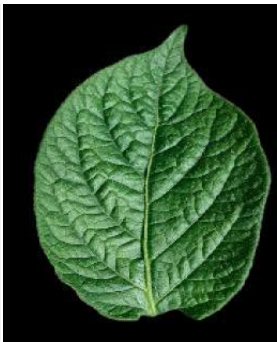

(c)

Figure 9 The first four rows show the apple leaves (scab, black rot, cedar apple rust and healthy), the fifth and sixth rows show the cherry leaves (healthy and powdery mildew). (a) original images, (b) Binary masked images, (c) Final masked images. 
Multiple Plant Leaf Disease Classification using Densenet-121 Architecture
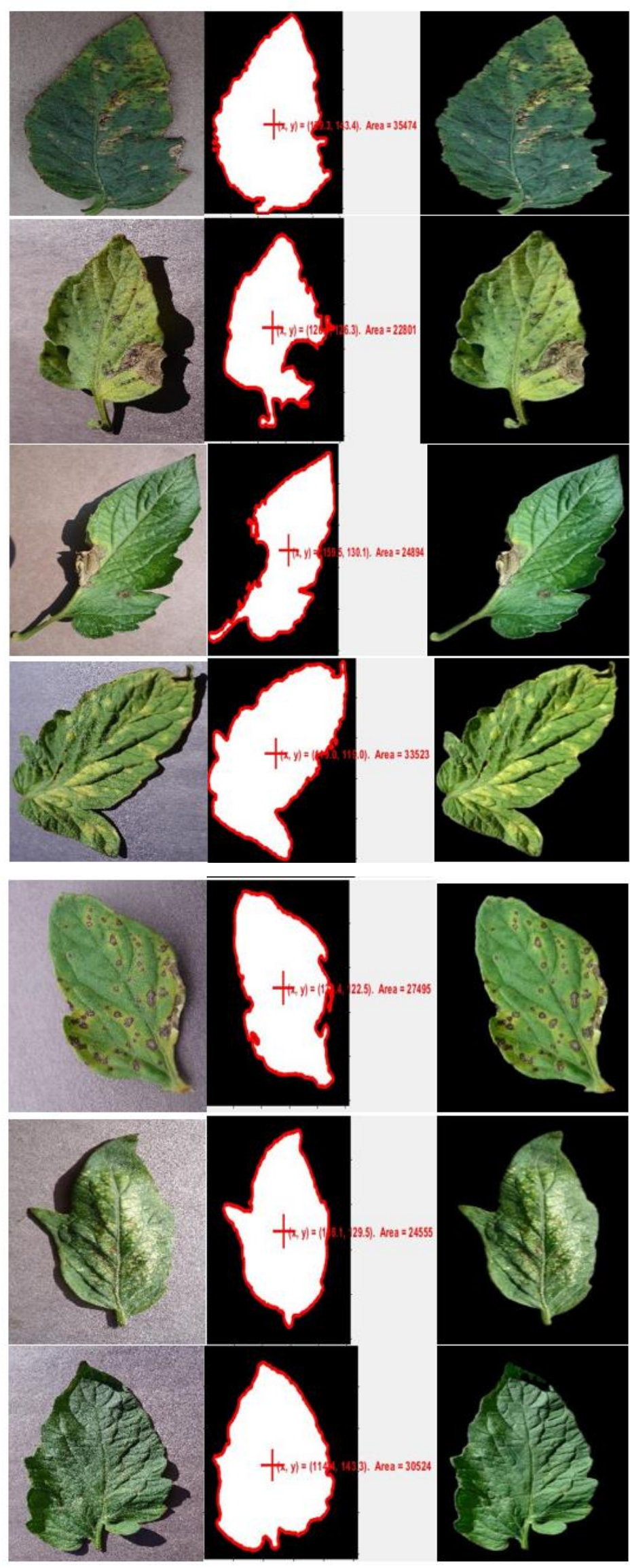


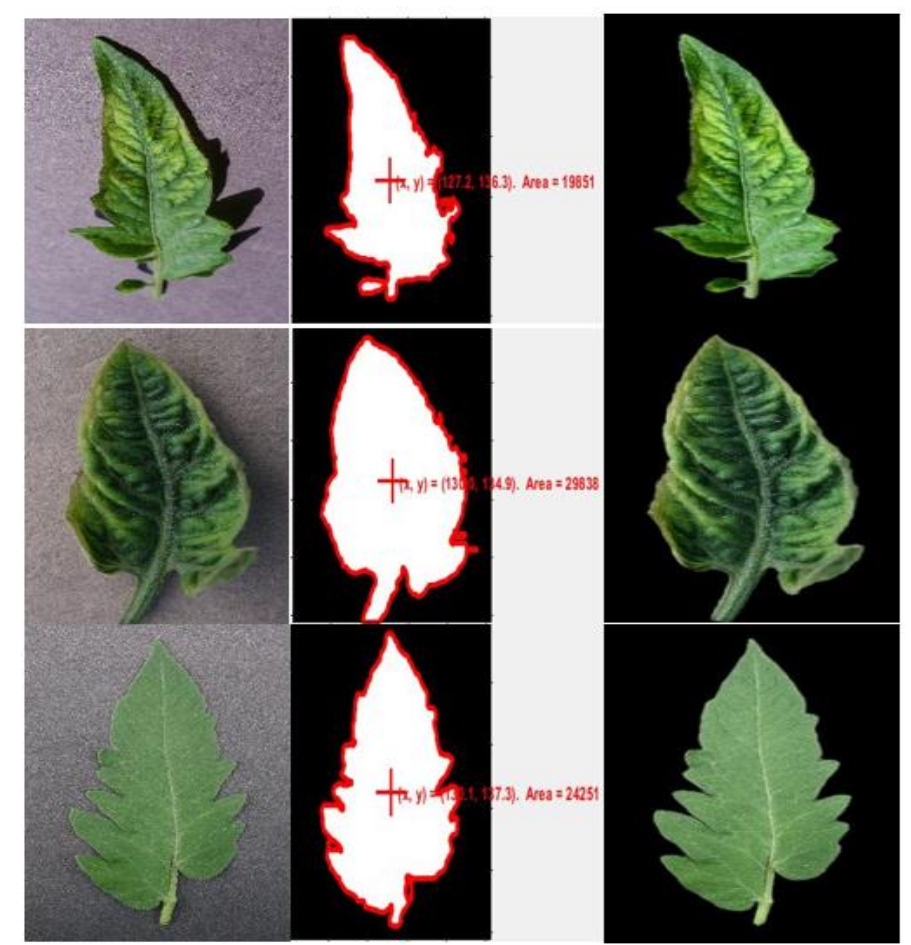

(a)

(b)

(c)

Figure 10 The Ten rows show the tomato leaves (bacterial spot, early blight, late blight, leaf mold, septoria leaf spot, spider mites, target spot, mosaic virus, yellow curl virus and healthy). (a) original images, (b) Binary masked images, (c) Final masked images.

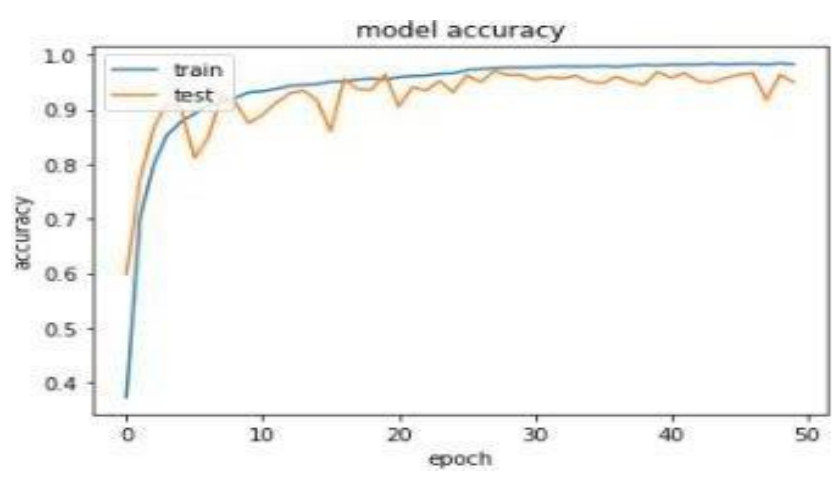

Figure 11 (a)

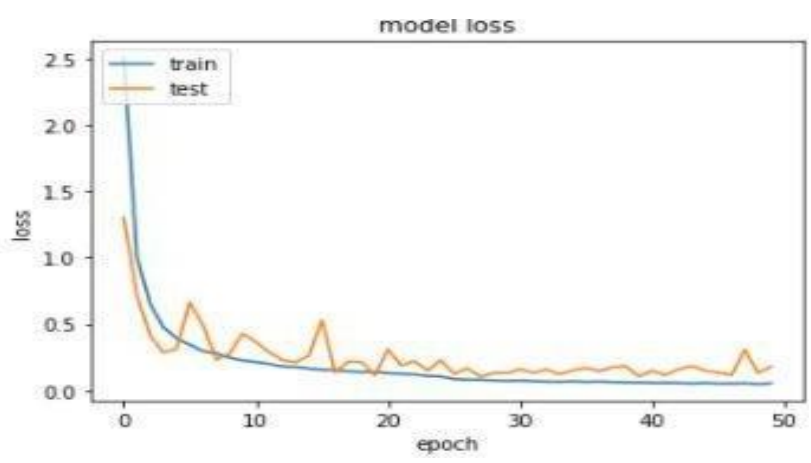

Figure 11: (b)

Figure 11 Shows the relation between number of iteration and average loss during training. Y- axis represents the average a) and loss (b) respectively and $\mathrm{X}$-axis represents the number of epochs. The model is trained up to 21850 iterations with average 94.96 and loss of 0.0634 . 


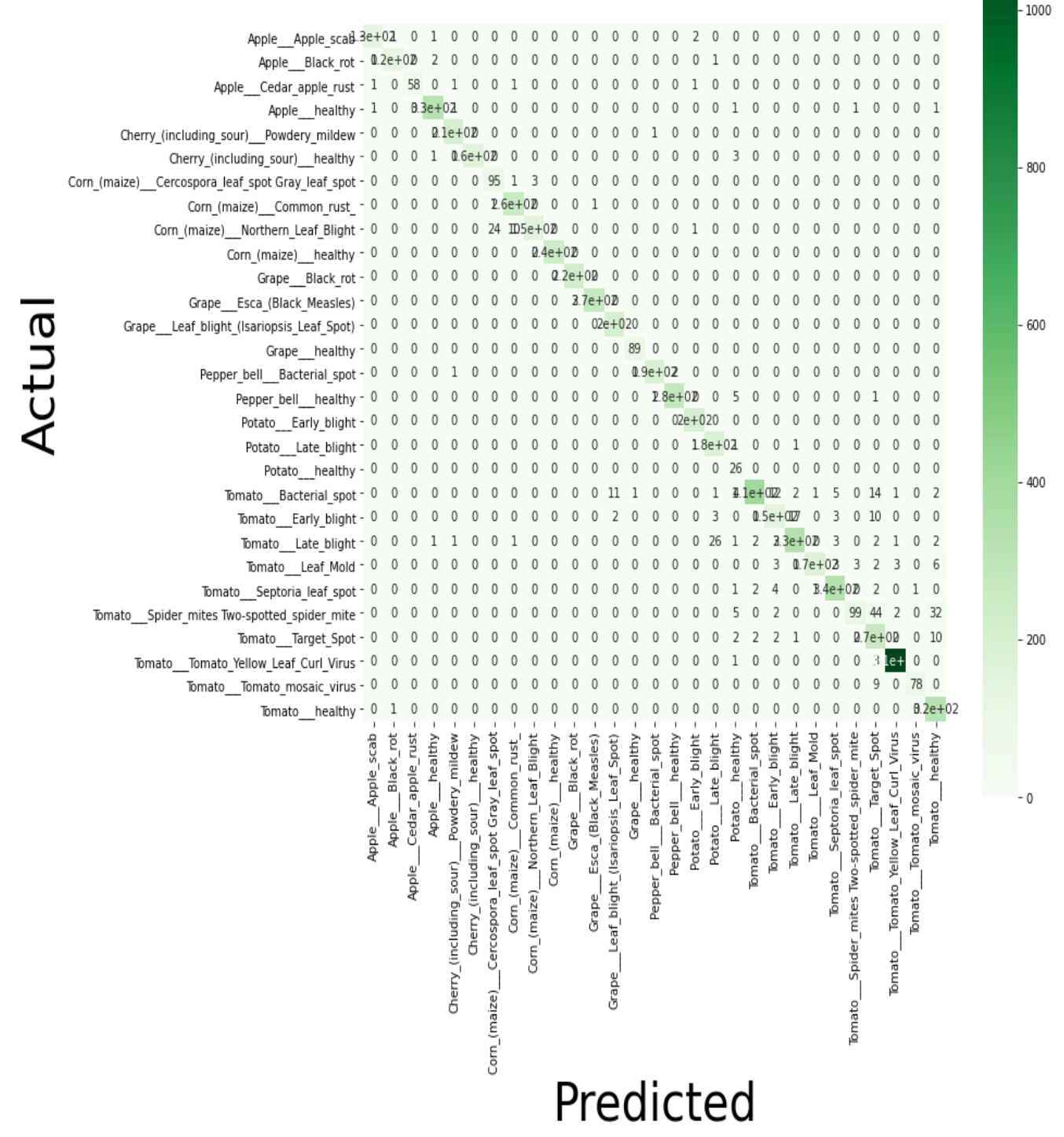

Figure 12 above shows the confusion matrix generated to show the statistical values for the performance of the neural network. The model has been trained ideal result i.e. to have a diagonal matrix plotted between the actual and the predicted results. The false predicted images are seen more in the tomato crop region, than compared to the rest of fields.

The detailed comparison of the proposed classification system with the state of art methods is given in below Table.3. The classification accuracy performances of different state of art methods IRPDD, MLPDD, and ACDMT are 72.2\%, 90.7\%, and 85.4\%, respectively for apple leaves image dataset. The KMCSAM and MLLFC methods show accuracies of $79.2 \%$ and $85.9 \%$ for apple dataset. The GAFADC method gave an accuracy of $85.7 \%, 82.5 \%, 80.5 \%, 86.8$, $81.6 \%, 81.0 \%$, and $77.2 \%$, for apple, cherry, corn, grape, pepper, potato, and tomato datasets, respectively. The proposed method offers an accuracy of $99.21 \%, 100 \%, 94.81 \%, 93.7 \%$, $96.1 \%, 91.9 \%$, and $88.2 \%$ for apple, cherry, corn, grape, pepper, potato and tomato datasets, respectively. The average accuracy of the feature set based multiple disease classifier (FSMDC) [18] is $93.2 \%$. Overall average we get $98.23 \%$ which outperforms the existing proposed methods. This theoretical average is calculated by averaging each plants' accuracy of detecting the respective classes under them. Also, practically i.e running all 29 labels of different classes of plants given to the model for classification we achieved $94.96 \%$ accuracy. 
Aswin Vellaichamy S, Akshay Swaminathan, C Varun and Kalaivani S

Table 3 Accuracies achieved for the plant disease classification

$\begin{array}{ccccccccccc}\text { Crop Name } & \text { Proposed } & \text { FSMDC } & \text { IRPDD } & \text { ACDMT } & \text { KMCSAT } & \text { MLLFC } & \text { GAFADC } & \text { MLPDD } \\ \text { Apple } & 99.21 & 94.8 & 77.2 & 85.4 & 79.2 & 85.9 & 85.7 & 90.7 \\ \text { Cherry } & 100 & 96.4 & 82.8 & 83.1 & 85.1 & 87.9 & 82.5 & 86.3 \\ \text { Corn } & 94.81 & 91.9 & 82.4 & 78 & 85.1 & 81.4 & 80.5 & 81.3 \\ \text { Grape } & 99.75 & 93.7 & 78.2 & 76.4 & 80.2 & 82.1 & 86.8 & 82.9 \\ \text { Pepper-bell } & 99.6 & 96.1 & 87.9 & 82.2 & 84.1 & 85 & 89.6 & 87.4 \\ \text { Potato } & 99.54 & 91.9 & 87 & 78.4 & 85.6 & 83.6 & 81 & 81.8 \\ \text { Tomato } & 94.69 & 88.2 & 80.1 & 77.6 & 84.7 & 78.4 & 77.2 & 81\end{array}$

\section{TESTING}

We have created a Web-App for testing our trained model. The figure 13 below is the homepage of our website (beta version). It consists of navbar for the users to navigate through the website.
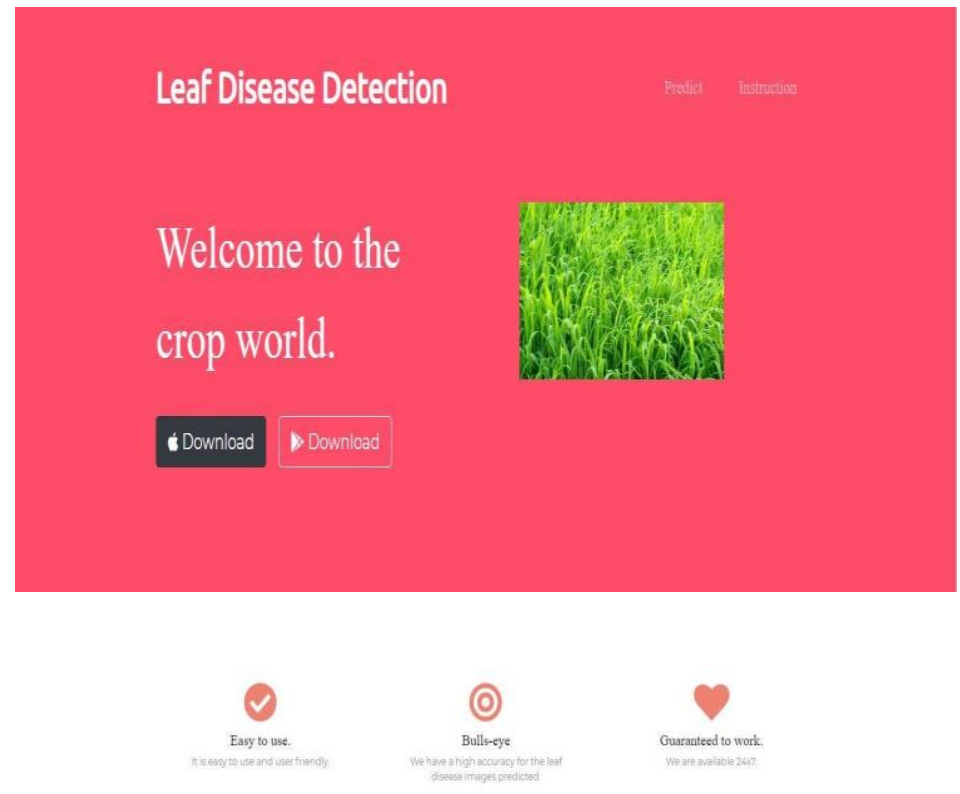

This app can be used for detecting leaf diseases of Tomato,

Potato, Pepper-bell, Maize, Apple, Grape and Cherry plants.

purpesese ramen

Figure 14 above consists of the testimonial section for the users to understand about the website and how it functions 


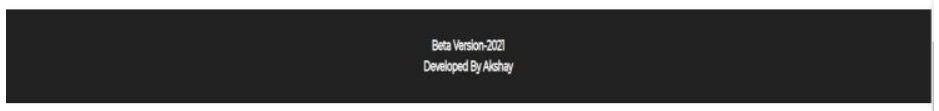

Figure 15 above is the part where the user can upload the image of the leaf to check which disease the plant has been infected

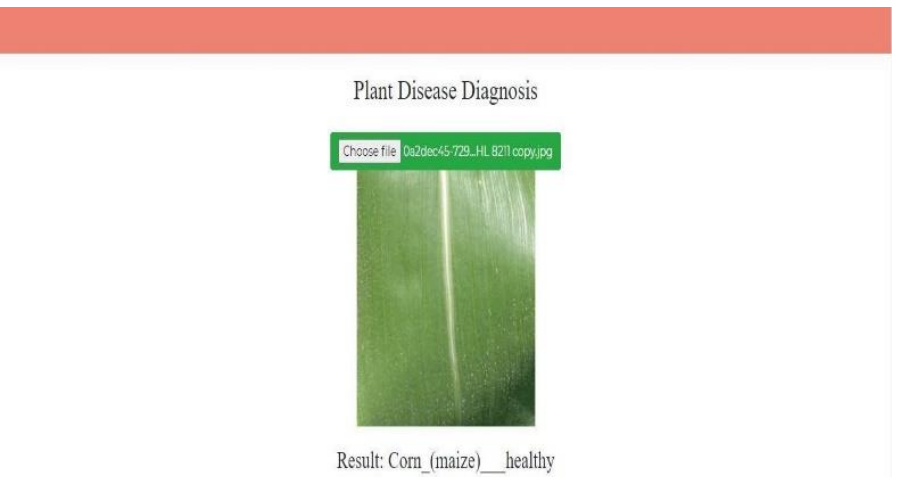

Figure 16

As soon as the user uploads the image, the user should click the predict button to get the desired results. The figure 16 above shows the example of detecting a corn leaf (healthy) plant

\section{CONCLUSION}

The detection of crop diseases can be determined by observing the structure deformation of leaf images. These features are given more priority by suppressing the background for more efficient classification of diseases. Firstly, we change the color form of the acquired image and binary mask it by thresholding, and apply it to the image to remove background details. The performance measure of the proposed work is analyzed through Plant-Village datasets of apple, cherry, corn, grape, bell pepper, potato, and tomato leaf images using DCNN model. The proposed work has shown an average accuracy of $98.23 \%$ theoretically and practically $94.96 \%$ outperforming the previously proposed state of art techniques.

\section{FUTURE SCOPE}

Our model can be extended to classify various other plant diseases like paddy crop, barley, lemon etc. with proper image dataset or can have improved accuracies by expanding the dataset to real-time images. As for the website, we have just established a beta version, this can be 
improved a lot more by deploying it in real time servers which would in turn improve our accuracy of detecting diseases in crops. An app would be a better work than of ours.

\section{REFERENCES}

[1] Anami, Basavaraj S., Naveen N. Malvade, and Surendra Palaiah. "Classification of yield affecting biotic and abiotic paddy crop stresses using field images." Information Processing in Agriculture 7, no. 2 (2020): pp 272-285.

[2] Muppala, Chiranjeevi, and Velmathi Guruviah. "Detection of leaf folder and yellow stemborer moths in the paddy field using deep neural network with search and rescue optimization." Information Processing in Agriculture (2020).

[3] Sethy, Prabira Kumar, Nalini KantaBarpanda, Amiya Kumar Rath, and Santi Kumari Behera. "Image Processing Techniques for Diagnosing Rice Plant Disease: A Survey." Procedia Computer Science 167 (2020): pp 516-530.

[4] Ramesh, S., and D. Vydeki. "Recognition and classification of paddy leaf diseases using Optimized Deep Neural network with Jaya algorithm." Information processing in agriculture 7, no. 2 (2020): pp 249-260.

[5] Khan, Saiqa, and Meera Narvekar. "Novel fusion of color balancing and supe rpixel based approach for detection of tomato plant diseases in natural complex environment." Journal of King Saud University-Computer and Information Sciences (2020).

[6] Mathew, Deepthy, C. Sathish Kumar, and K. Anita Cherian. "Foliar fungal disease classification in banana plants using elliptical local binary pattern on multiresolution dual tree complex wavelet transform domain." Information Processing in agriculture (2020).

[7] Khan MA, Lali MIU, Sharif M, et al. An Optimized Method for Segmentation and Classification of Apple Diseases Based on Strong Correlation and Genetic Algorithm Based Feature Selection. IEEE Access. 2019March;7:46261-46277.

[8] Sharma, Parul, Yash Paul Singh Berwal, and Wiqas Ghai. "Performance analysis of deep learning CNN models for disease detection in plants using image segmentation." Information Processing in Agriculture 7, no. 4 (2020): pp 566-574

[9] Bakar, MN Abu, A. H. Abdullah, N. Abdul Rahim, H. Yazid, S. N. Misman, and M. J. Masnan. "Rice leaf blast disease detection using multi-level colour image thresholding. "Journal of Telecommunication, Electronic and Computer Engineering (JTEC) 10, no. 1-15 (2018): 1-6.

[10] Rahman, Chowdhury R., Preetom S. Arko, Mohammed E. Ali, Mohammad A. Iqbal Khan, Sajid H. Apon, Farzana Nowrin, and AbuWasif. "Identification and recognition of rice diseases and pests using convolutional neural networks." Biosystems Engineering 194 (2020): pp112-120

[11] Mishra, S., Sachan, R. and Rajpal, D., 2020. Deep Convolutional Neural Network based Detection System for Real-time Corn Plant Disease Recognition. Procedia Computer Science, 167, pp.2003-2010.

[12] Afonso, M., Blok, P.M., Polder, G., van der Wolf, J.M. and Kamp, J., 2019. Blackleg Detection in Potato Plants using Convolutional Neural Networks. IFAC-Papers OnLine, 52(30), pp.6-11.

[13] Tiwari, D., Ashish, M., Gangwar, N., Sharma, A., Patel, S. and Bhardwaj, S., 2020, May. Potato leaf diseases detection using deep learning. In 2020 4th International Conference on Intelligent Computing and Control Systems (ICICCS) pp. 461-466. IEEE.

[14] Sibiya, M. and Sumbwanyambe, M., 2019. A computational procedure for the recognition and classification of maize leaf diseases out of healthy leaves using convolutional neural networ Agri Engineering, 1(1), pp.119-131. 
[15] Vetal, S. and Khule, R.S., 2017. Tomato plant disease detection using image processing. International Journal of Advanced Research in Computer and Communication Engineering, 6(6), pp.293-297.

[16] Adhikari, S., Shrestha, B., Baiju, B. and Saban, K.C., 2018. Tomato plant diseases detection system using image processing. In 1st KEC Conference on Engineering and Technology, Lalitpur Vol. 1, pp. 81-86.

[17] Athanikar, G. and Badar, P., 2016. Potato leaf diseases detection and classification system. International Journal of Computer Science and Mobile Computing, 5(2), pp.76-88.

[18] Kurmi, Y. and Gangwar, S., 2021. A Leaf Image Localization based Algorithm for Different Crops Disease Classification. Information Processing in Agriculture.

[19] Iyyanar, P., Priya, R., Saranya, E. and Sindhuja, S., PLANT DISEASE PREDICTION.

[20] Jadhav, M.V.S., 2021. Development and Advancement in Image Processing Technique for Detection of Various Diseases in Fruit \& Vegetable Plants: A Review.

[21] Tummala, S., 2021. Classification of Multi Diseases in Apple Plant Leaves

[22] Ghorai, A.K., Mukhopadhyay, S., Kundu, S., Mandal, S.N., Barman, A.R., De Roy, M., Jash, S. and Dutta, S., Image Processing Based Detection of Diseases and Nutrient Deficiencies in Plants.

[23] Patil, J., 2021. Pomegranate Fruit Diseases Detection Using Image Processing Techniques: A Review. Information Technology In Industry, 9(2), pp.115-120.

[24] Kartikeyan, P. and Shrivastava, G., Review on Emerging Trends in Detection of Plant Diseases using Image Processing with Machine Learning. International Journal of Computer Applications, 975, p.8887.

[25] Zarlis, M. and Sihombing, P., 2021, February. Optimization of the CNN model for smart agriculture. In IOP Conference Series: Materials Science and Engineering (Vol. 1088, No. 1, p. 012029). IOP Publishing.

[26] Huang, G., Liu, Z., Van Der Maaten, L. and Weinberger, K.Q., 2017. Densely connected convolutional networks. In Proceedings of the IEEE conference on computer vision and pattern recognition (pp. 4700-4708).

[27] Zhang, Y., Tian, Y., Kong, Y., Zhong, B. and Fu, Y., 2018. Residual dense network for image super- resolution. In Proceedings of the IEEE conference on computer vision and pattern recognition (pp. 2472-2481).

[28] Guo, Z., Zhang, Y., Teng, Z. and Lu, W., 2019. Densely connected graph convolutional networks for graph-to-sequence learning. Transactions of the Association for Computational Linguistics, 7, pp.297-312.

[29] Patil P, Yaligar N, M MS. Comparison of Performance of Classifiers -SVM, RF and ANN in Potato Blight Disease Detection Using Leaf Images. In: IEEE Intern. Conf. Compu. Intell. and Comput. Research (ICCIC);2017. p. 1-5.

[30] Qin F, Liu D, Sun B, et al. Identification of Alfalfa Leaf Diseases Using Image Recognition Technology. PLOS ONE. 2016 12;11(12):1-26.

[31] Kaur S, Pandey S, Goel S. Semi-automatic leaf disease detection and classification system for soybean culture. IET Image Processing. 2018May;12(6):1038-1048.

[32] Hughes DP, Salathe M. An open access repository of images on plant health for mobile disease diagnosis 